\title{
MedienPädagogik
}

Zeitschrift für Theorie und Praxis der Medienbildung

\section{Editorial: Virtualität und E-Learning}

Horst Dichanz

Wer Anfang April den Weltkongress des ICDE in Düsseldorf besuchte, der unter dem Titel veranstaltet wurde, war von der Fülle der Veranstaltungen überrascht, die sich auf die eine oder andere Weise aus unterschiedlichster Sicht mit Fragen von Virtuellen Lernwelten und den Möglichkeiten des E-Learning befassten. Mindestens ebenso schwer zu überblicken waren die zahlreichen Produkte, die von Firmen aus zahlreichen Ländern für Zwecke der Gestaltung von virtuellen Lernwelten und zur Durchführung von E-Learning-Veranstaltungen angeboten wurden. Diese E-Learning-Produkte reichten von simplen Datenbanken verschiedenster Fächer über Lern- und Kommunikationsplattformen bis hin zu komplexen Oberflächen oder Rahmenprogrammen zur Konstruktion eigener Lernsoftware. - Über solide, methodisch nachprüfbare Erfolgsuntersuchungen konnten allerdings nur wenige berichten.

Die Szene im Internet, das man auf «Virtualiät» und «E-Learning» durchforstet, ist kaum anders: Dutzende von Firmen und Instituten bieten «E-Learning»-Produkte an. Ohne im Detail zu beschreiben, was an ihrem Produkt nun «E-Learning» ist. In den meisten Fällen stellt sich heraus, dass es sich dabei um elektronisch gespeicherten, bearbeitbaren und/oder gewinnbaren Lernstoff handelt, dessen Lernnutzen zweifelsfrei unterstellt wird.

Ein zweiter Schwachpunkt: Was konkret unter «Lernen» mithilfe elektronischer Medien gemeint ist, was sich die Produzenten, Autoren u.ä. darunter vorstellen, wird noch seltener thematisiert. Gleichwohl ist der Markt in allen Bildungsbereichen riesig, er wird als expandierend eingeschätzt, in Verbindung mit den elektronischen Medien wird gelegentlich sogar der Bildungsmarkt als ein Wachstumsmarkt charakterisiert. Viel Grund zu Optimismus, noch mehr Grund zu detaillierten Rückfragen!

Doch sind Arbeiten, genaue Untersuchungen zu den Komplexen «Virtualität» und «E-Learning» noch mit der Lupe zu suchen. Es gehört zweifellos zu den Aufgaben einer Zeitschrift «medienpaedagogik», die sich noch dazu ihren Lesern online präsentiert, forschungsrelevante Fragen in Verbindung mit Virtualität und E-Learning aufzuwerfen, Erfahrungen aus dem Feld aufzugreifen und auszuwerten und weiterführende Fragen zu entwickeln, die mit diesen beiden Komplexen zusammenhängen. Dies wird in dieser Ausgabe versucht.

Dabei stehen erste Erfahrungsberichte neben tastenden Forschungsansätzen und theoretischen Analysen oder dem Versuch, aus bisherigen Erfahrungen erste didaktische Hinweise zu gewinnen.

So stellt Horst Heidbrink schon einen zusammengefassten Erfahrungsbericht aus mehreren virtuellen Seminaren vor, die im Institut für Psychologie an der Fernuniversität mit insgesamt ca. 200 Teilnehmern durchgeführt wurden. Seine Bezüge

Dichanz, Horst. 2001. «Editorial: Virtualität und E-Learning». MedienPädagogik 2, (April), i-ii. https://doi.org/10.21240/mpaed/02/2001.04.18.X. 
zur einschlägigen Literatur bestätigen, wie jung diese Erfahrungen und Reflexionen darüber sind. Ina Siekmann geht auf einen ähnlichen Erfahrungshintergrund ein, thematisiert jedoch vor allem Fragen emotionaler Relevanz, die bei vielen bisherigen Betrachtungen von virtuellen Veranstaltungen völlig unbeachtet blieben. Zwar reicht ihr Material noch nicht für eine weitergehende systematische Analyse - dafür ist die Basis zu schmal -, es macht aber neugierig auf Beobachtungen und Analysen, die sich dezidiert dem Komplex der Emotionalität in virtuellen Lernwelten zuwenden. Soviel scheint sicher: Emotionales Verhalten tritt auch dort auf, die Formen scheinen aber vielfältiger und anders zu sein als bei direktem kommunikativen Kontakt. Eine der ganz wenigen differenziert empirischen Erhebung aus viruteller Lernarbeit stellt der Beitrag von Marion Degenhardt dar, die mithilfe der Analyse von Logfiles der tatsächlichen Computernutzung eines Sampels von Schülerinnen und Schülern nachgeht. Außer aus dringendem empirischen Forschungsbedarf ist der Beitrag auch aus pädagogisch-didaktischer Sicht bedeutsam. Zum einen erhellt er unsere Vorstellungen davon, wie Schülerinnen und Schüler tatsächlich mit Lernsoftware umgehen, zum anderen liefert er damit wichtige Hinweise auf die künftige Gestaltung von Lernsoftware und gibt eine methodische Grundlage, hierfür Kriterien zu entwickeln. Hofffentlich regt er auch andere Forscher an, das Fundament des empirisch gesicherten Wissens über E-Learning durch weitere Untersuchungen zu verbreitern. Die Verbindung von dieser Untersuchungen zu einem Versuch, unser noch dürftiges Wissen über die Nutzung von elektronisch-virtuellen Lernwelten pädagogisch zu nutzen und daraus methodisch-didaktische Konsequenzen zu ziehen, unternimmt Peter Kührt in seinem Beitrag über Future Guide, einem didaktischen Gestaltungsprinzip auf der Basis handlungsorientierter Lernumgebungen.

Horst Dichanz und Annette Ernst nehmen abschließend noch einmal die Diskussion um das E-Learing auf und versuchen, aus der Fülle und Unübersichtlichkeit der Begriffsverwendungen eine Klarstellung der Begriffe. Sie untersuchen mit Bezug auf eine konstruktivistische Interpretation von Lernprozessen die realen Möglichkeiten der Nutzung von elektronischen Lernhilfen und -environments und dokumentieren sie abschließend mit einem Projektdesign eines gerade begonnen Projektes zur Lehrerfortbildung.

Diese Ausgabe der Zeitschrift MedienPädagogik zielt darauf, in Zusammenhang mit den Begriffen, Produkten und Projektentwürfen zu virtuellen Lernumwelten die Möglichkeiten und Grenzen des E-Learning kritisch zu überprüfen, für eine realistische Betrachtungsweise zu sensibilisieren und Forschungsbedarf zu artikulieren. Vielleicht wird es dann in einigen Jahren möglich sein, erste Forschungsergebnisse in systematisierter Form vorzulegen. 